\title{
HYMAD: Hybrid DTN-MANET Routing for Dense and Highly Dynamic Wireless Networks ${ }^{\text {th }}$
}

\author{
John Whitbeck ${ }^{\mathrm{a}, \mathrm{b}}$, Vania Conan ${ }^{\mathrm{a}}$ \\ ${ }^{a}$ Thalès Communications \\ 160 bd de Valmy - 92704 COLOMBES Cedex - France \\ ${ }^{b}$ UPMC Paris Universitas \\ Laboratoire d'Informatique de Paris 6 \\ 104 avenue du Président Kennedy - 75016 Paris - France
}

\begin{abstract}
Delay/Disruption-Tolerant Network (DTN) protocols typically address sparse intermittently connected networks whereas Mobile Ad-hoc Network (MANET) protocols address the fairly stable and fully connected ones. But many intermediate situations may occur on mobility dynamics or radio link instability. In such cases, where the network frequently splits into evolving connected groups, none of the conventional routing paradigms (DTN or MANET) are fully satisfactory. In this paper we propose HYMAD, a Hybrid DTN-MANET routing protocol which uses DTN between disjoint groups of nodes while using MANET routing within these groups. HYMAD is fully decentralized and only makes use of topological information exchanges between the nodes. The strength of HYMAD lies in its ability to adapt to the changing connectivity patterns of the network. We evaluate the scheme in simulation by replaying synthetic and real life mobility traces which exhibit a broad range of connectivity dynamics. The results show that HYMAD introduces limited overhead and outperforms the multi-copy Spray-and-Wait DTN routing protocol it extends, both in terms of delivery ratio and delay. This hybrid DTN-MANET approach offers a promising venue for the delivery of elastic data in mobile ad-hoc networks as it retains the resilience of a pure DTN protocol while significantly improving performance.
\end{abstract}

Keywords: Delay Tolerant Networks, Routing

\footnotetext{
This article is an expanded version of work presented at the IEEE WoWMoM Workshop on Autonomic and Opportunistic Communications (AOC 2009) [1]. This paper presents allnew results based on a full implementation of HYMAD in the ONE DTN Simulator [2], as well as a more detailed evaluation of HYMAD in terms of network dynamics, density, and overhead.

Email addresses: john.whitbeck@lip6.fr (John Whitbeck), vania.conan@fr.thalesgroup.com (Vania Conan)
} 


\section{Introduction}

Wireless ad-hoc networking has emerged over the past decades at the intersection of personal computing, cellular telephony, and the Internet. It is best suited for use in situations where an infrastructure is not available, or too costly to deploy. In a wireless ad-hoc network, radio equipped devices (laptops, smart phones, sensors, etc.) cooperate in a distributed manner to provide the necessary network functionality in the absence of a fixed infrastructure. Applications include for example emergency and rescue operations, conference or campus settings, body area and personal networks, or vehicular networks.

Node mobility has been acknowledged as one of the key challenges of adhoc networking with direct impact on protocol performance. Early models, such as Random Walk, Random Direction and Random Waypoint, consider nodes moving on a free-space planar surface. More refined characterization and modeling have been derived for pedestrian applications [3, 44 and for vehicular mobility [5].

The measurements and observations of mobility patterns, ranging from students on a campus to pedestrians or taxi-cabs in a city, have pushed the community to also consider extremely sparse networks, or very dynamic mobility conditions, e.g. often observed in the vehicular setting, where end-to-end paths between any pair of nodes may seldom exist [6, 7].

For such sparse or dynamic cases, the Delay/Disruption-Tolerant Network (DTN) 8] paradigm uses node mobility to its advantage while compromising on message delivery delays [9]. Message forwarding decisions are made on a per-encounter basis, for example by using utility functions based on aggregating statistics on node meeting probabilities [10, 11, 12]. At any given time, a node's vision of the network topology is limited to its current neighbors, whereas conventional Mobile Ad-hoc Network (MANET) routing schemes have either complete (in the proactive case) or at least partial (in the reactive case) knowledge of the actual network topology.

But even in the extreme case of sparse of highly mobile networks there are situations where the network is sufficiently dense and well connected to temporarily provide end-to-end connectivity between a significant subset of its nodes.

These nodes may even form small islands of stability. Using MANET principles within such islands can bring great improvements. Indeed, it considerably increases each node's information of its local topology, thus leading to better forwarding decisions. When high mobility rates and more generally high link instabilities reduce route life-times and threaten network-wide end-to-end connectivity, a MANET routing protocol can still succeed locally even if it fails globally.

In this paper we propose HYMAD, a Hybrid DTN-MANET routing protocol. HYMAD combines techniques from both traditional ad-hoc routing and DTN approaches. HYMAD periodically scans for network topology changes and builds temporary disjoint groups of connected nodes. Intra-group delivery is performed by a conventional ad-hoc routing protocol and inter-group delivery 


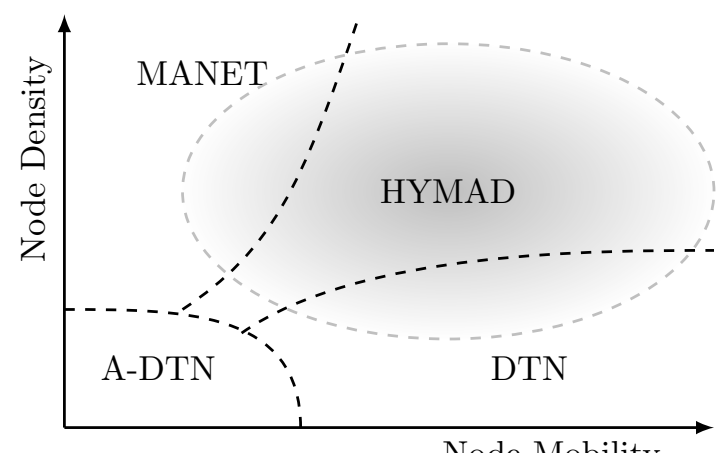

Figure 1: Mobility vs Density: when different paradigms apply

by a DTN protocol.

HYMAD constantly adapts to the dynamics of the wireless ad-hoc network using only topological information. As in traditional ad-hoc routing, no extra information on geographical location or social community membership is required. It does not rely on a priori knowledge of connectivity patterns or inter-meeting times. This makes HYMAD amenable to implementation in a DTN stack or ad-hoc routing protocol [13]. In a dense network, HYMAD can function similarly to a traditional MANET protocol. In the other extreme case of very sparse connectivity (where only two nodes can be in contact at any time) each node is a group on its own and HYMAD behaves like a classical DTN routing protocol. In any other intermediate case its hybrid nature takes over.

We implemented the HYMAD hybrid approach in the ONE DTN simulator [2] with a self-stabilizing group service [14, 15] and the multi-copy Sprayand-Wait protocol as the DTN routing scheme [16.

We evaluated the scheme by performing simulation runs both on synthetic Random Waypoint mobility traces and on the real-life Rollernet data set [17], an example of a highly dynamic ad-hoc network.

In the next section, we further describe how this hybrid approach positions itself compared to existing DTN and MANET approaches. In section 3, we describe the HYMAD routing protocol principles. We explain how nodes can agree on forming disjoint groups and how such groups rather than individual nodes can be used as the basis for DTN routing. We then evaluate the scheme both on synthetic mobility models and on a real data set, the Rollernet experiment, in section 4 The results show that HYMAD outperforms the multi-copy Spray-and-Wait DTN routing protocol it extends, both in terms of delivery ratio and delay.

\section{Routing in a mobile wireless network}

Mobile wireless ad-hoc networks were first studied under the assumptions of moderate node mobility and sufficient density to ensure end-to-end connectiv- 
ity. Both conditions are necessary for traditional MANET approaches, be they proactive or reactive.

Recently, there has been an effort to classify the various types of mobile ad-hoc networks [18]. One can characterize the relevant routing paradigms in mobile wireless networks along the two main parameters of node density and node mobility. In Fig. 1, which maps the different routing approaches on the bidimensional mobile wireless network space, traditional MANET routing appears in the top left corner.

When the density of nodes diminishes, end-to-end connectivity can disappear. In such sparse networks, nodes have very few, if any, neighbors within their transmission ranges. The topology eventually splits into several noncommunicating connected components. This is typically the realm of Delay Tolerant Networking which one can further subdivide in two [19]: the Assisted DTNs (A-DTN), in case of low mobility of nodes, or Unassisted DTNs (U-DTN) where mobility is high. The latter corresponds to traditional DTN scenarios.

Routing in A-DTNs typically involves special mobile nodes, known as message ferries or data mules, which relay the messages between the separate connected components [20, 21]. The packet-switching method of MANETs is replaced with a store-and-forward approach.

When the mobility in sparse networks increases, mobile nodes begin to meet others. This is the traditional DTN scenario, where nodes forward one or more copies of a given message until it reaches its destination. There are many strategies for optimizing the forwarding decision. The most straightforward approaches, such as Epidemic or Spray-and-Wait [16] do not require nodes to acquire information on the others' positions, movements or trajectories. More elaborate schemes involve a utility function where each node collects direct and indirect knowledge of other nodes' meeting probabilities. They require a certain learning period to aggregate statistics before making good forwarding decisions. For example, Lindgren et al. [10] use past encounters to predict the probability of meeting a node again while Daly et al. 11] use local estimates of betweeness and similarity.

In dense networks, conventional MANET protocols start to break down under high mobility down even if the network is almost always fully connected. Indeed the sheer instability of the links would result in a deluge of topology updates in the proactive case and route error and new route requests messages in the reactive case. DTNs protocols on the other hand can handle high mobility regardless of the density of the network. However by narrowly focusing on per-encounter events, they ignore a lot of available information. For example, simply asking nodes to regularly broadcast a list of their neighbors would give each node a picture of its two-hop neighborhood even under high mobility. Repeat this once and everyone knows their three hop neighborhood. A node may therefore have a topology "knowledge horizon" which determines how far into the real topology a node can "see". The more extreme the mobility, the shorter the "horizon".

The Hybrid DTN-MANET approach that we advocate in this paper aims at filling the gap for efficient routing in highly connected and highly mobile 
Table 1: HYMAD groups vs. DTN nodes

\begin{tabular}{ll} 
DTN & HYMAD \\
\hline Node & Group of nodes \\
\hline $\begin{array}{l}\text { A node has mes- } \\
\text { sage } m\end{array}$ & $\begin{array}{l}\text { One node in the group has message } \\
m \text { and all other nodes in the group } \\
\text { know that. }\end{array}$ \\
\hline Two nodes meet & $\begin{array}{l}\text { Two disjoint groups become con- } \\
\text { nected. }\end{array}$ \\
\hline
\end{tabular}

networks, which have so far, to the best of our knowledge, received little attention. Hybrid DTN-MANET routing, like the HYMAD protocol that we describe below, combines the resilience of DTNs with the greater knowledge of local network topology provided by a MANET protocol. It adapts naturally to the dynamics of the network and its applicability spans a large spectrum of the mobile wireless network space.

\section{The HYMAD protocol}

\subsection{Overview}

The core idea in HYMAD is to use whole groups of nodes instead of individual nodes as the focus of a DTN protocol. The analogy is detailed in Table 1

Each node $u$ regularly broadcasts, not necessarily with the same frequency, two control messages:

1. An "enhanced distance vector" for all members of its group.

2. A list of the messages held by members of its group, with their respective destinations, custodians, and number of copies.

The first message enables the use of an intra-group distance vector routing protocol. In this paper's implementation, the actual transmitted distance vector is slightly modified with a node marking mechanism for the distributed network partitioning algorithm (Section 3.2). Furthermore, each node can be tagged as a border node (i.e. in contact with other groups). We will refer to this list as the Group algorithm message.

The second message is necessary for the inter-group routing protocol. The message list allows a group to agree on what messages it carries, and, for each message, which node (hereafter call the message's custodian) specifically holds it. The border node tags give every group member an estimation of the number of outgoing connections to neighboring groups. We will refer to this list as the Messages-in-group list.

As in traditional distance vector algorithms, the number of iterative broadcasts necessary for all members of a group to agree on this information is equal to the diameter of the group.

HYMAD then uses a DTN protocol to transfer messages between groups. The approach is generic and many existing DTN protocols could be employed. 


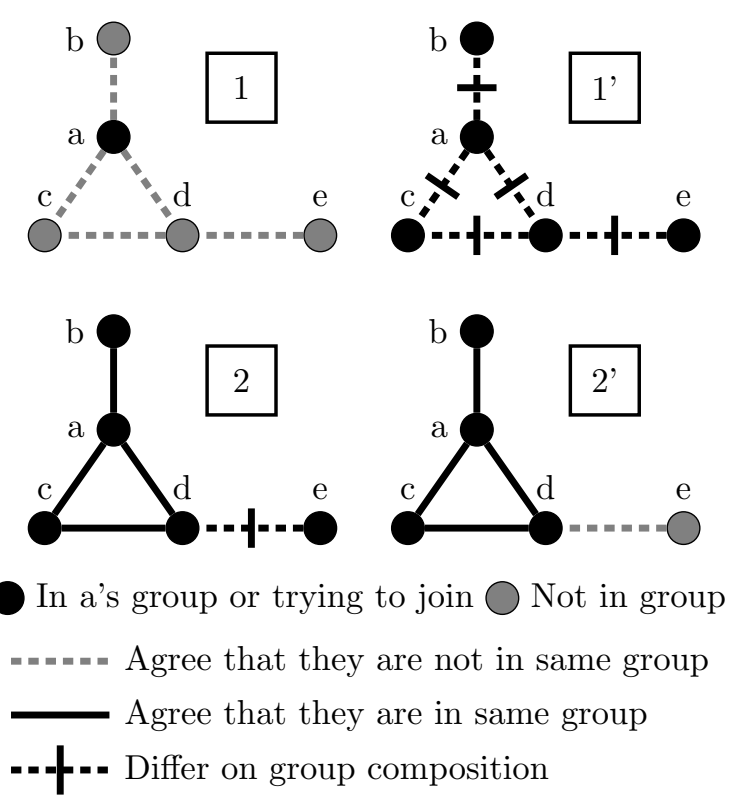

Figure 2: Self-stabilizing groups: convergence in two iterations

In this paper, Spray-and-wait [16] is used to forward messages between disjoint groups. As in Spray-and-Wait, the source of a message will create a certain number of copies of it. In HYMAD however, this source node is part of a group and copies of the message will be distributed among the adjacent groups instead of simply the nodes that the source encounters. If a group has more than one copy, it will, in turn, distribute extra copies to its other adjacent groups. If a group has just one copy it will wait until encountering the destination's group to transfer it. Once inside the destination's group, the intra-group routing protocol delivers the message to the destination.

\subsection{Intra-group routing}

In HYMAD, the intra-group routing is handled by a simple distance vector algorithm. The only difference is that if a next hop is no longer available, instead of discarding the message, the node takes custody of it and adds it to its list of held messages in order to advertise the fact to its group members.

That stills leaves open the question of how to partition the network into several disjoint groups of nodes using a simple distributed network partitioning algorithm. In our implementation, we chose to consider diameter-constrained groups. This is a particularly relevant metric because it preserves node equality and interchangeability. Indeed, another way of defining groups could have involved considering all the nodes within two hops, for example, of a given node. However this would mean that some nodes are somehow more important than others. The diameter parameter on the other hand does not refer to any par- 
ticular node and only makes sense within a connected subset of the nodes. It is therefore a natural candidate for defining disjoint groups of nodes.

A group will accept new members as long as its diameter is less than a predefined network-wide maximum diameter parameter $\left(D_{\max }\right)$. If a group's diameter expands due to internal link failure, then some members are excluded to satisfy the diameter constraint. Ducourthial et al. [15] propose a self-stabilizing, asynchronous distributed algorithm that achieves this using an $r$-operator on a slightly modified distance vector. This algorithm converges in $O\left(D_{\max }\right)$ iterations. The proof of self-stabilization using asynchronous message passing can be found in [14].

The main ideas behind group creation and modification are illustrated in Fig. 2 for a maximum diameter $D_{\max }=2$. In the first iteration, node $a$ begins by broadcasting the distance vector $(a: 0)$. Nodes $b, c$ and $d$ decide they want to join the group and broadcast $(b: 0, a: 1),(c: 0, a: 1)$, and $(d: 0, a: 1)$, respectively. After receiving the broadcast from $d$, node $e$ also decides that it wants to join the group and broadcasts $(e: 0, d: 1, a: 2)$ (or $(e: 0, d:$ $1, c: 1, a: 2)$ if $c$ spoke before $d$ ). In the second iteration, $a$ now broadcasts $(a: 0, b: 1, c: 1, d: 1), d$ realizes that the distance between $b$ and $e$ is greater than $D_{\max }$ and therefore chooses to exclude $e$ from the group and broadcasts $(d: 0, a: 1, c: 1, b: 2)$. Finally $e$ understands that it is not part of the group. After two iterations, the group has stabilized on $a, b, c, d$. Now lets suppose that at a later time the link between $a$ and $c$ goes down. Node $c$ now only receives the broadcasted distance vector $(d: 0, a: 1, b: 2)$ from $d$. It then understands that it is no longer part of the group. As is obvious from this example, a given topology can result in very different groups depending on the order in which the nodes speak.

Some looping problems can arise if two nodes simultaneously try to join a group that can only accept one of them. In order to avoid this, Ducourthial et al. suggest a priority mechanism, though refrain from actually detailing one [15]. In order to work, the priority function has to define a total order on the nodes. An interesting goal would be to ensure that a stable group cannot be split up when a new node, which happens to have priority over some group members, comes within range. We achieve this by defining each node's priority as either the time when it joined its current group or the current time if it is not in a group. We add to this a small value unique to each node that guarantees that a total ordering is defined at all times. These priority scores are attached to the Group algorithm message.

As in some MANET protocols, this algorithm is used in a proactive fashion where each node periodically runs the algorithm and broadcasts its modified distance vector. Group composition therefore changes in reaction to topology changes rather than routing needs. Unlike most MANET protocols, this information is never propagated beyond a group and its immediate neighbors. The size of the Messages-in-group lists is proportional to the number of nodes in a group, while the convergence time for all group information is in $O\left(D_{\max }\right)$. 


\subsection{Inter-group routing}

Border nodes take care of most of the inter-group DTN routing. Indeed, the periodic broadcast protocol described in 3.2 puts them in the unique position of knowing both the composition of two adjacent groups as well as the messages these hold. Border nodes may request a message's custodian to transfer one or more copies to it.

When a border node learns that its group has acquired copies of a message that a neighboring group does not possess, it has the following options:

- If the message's destination is in the neighboring group, request the message from its custodian and pass it on.

- If its group has more than one copy of the message, request $\max \left(1,\left\lceil\frac{n_{c}}{n_{b}+1}\right\rceil\right)$ copies from its custodian and pass them on. $\left(n_{c}\right.$ is the number of copies and $n_{b}$ the current number of border nodes in the group). The idea is to fairly spread a group's copies among its adjacent groups.

- Otherwise do nothing

Conversely, when a border node receives copies of a new message from an adjacent group it can either:

- If the destination is in its group, forward the message to it using the inter-group routing protocol.

- Otherwise, randomly select a group member to be the custodian for the copies. This is done to spread the burden over members of a group.

With this in place, when a node wants to send a message, it simply adds it to its own list of messages. Through the intra-routing protocol, in $O\left(D_{\max }\right)$ time, the group's border nodes will become aware of the new message and request copies to forward it on to the adjacent groups.

In order to achieve this, two types of control messages are needed: a Copy Request message to ask a node in a group to forward a copy, and a Reduce Number of Copies message to ask a node in a group to reduce the number of copies it currently holds. The latter message generates a response indicating how many copies many the node actually managed to remove. The importance of these two control messages is detailed in section 3.5.2 below, but it is important to stress that these messages are very small and their overhead is negligible compared to the overhead of the Group algorithm message and Messages-ingroup list that the nodes periodically broadcast.

\subsection{Example}

Fig. 3 details an example of HYMAD message forwarding. In this case, the HYMAD groups have stabilized into two disjoint groups (of diameter 2), a black one containing nodes A, B, C, and D, and a white one containing nodes F, E, and

G. Node $\mathrm{F}$ creates 5 copies of a message for node $\mathrm{C}$. It then adds the message to 

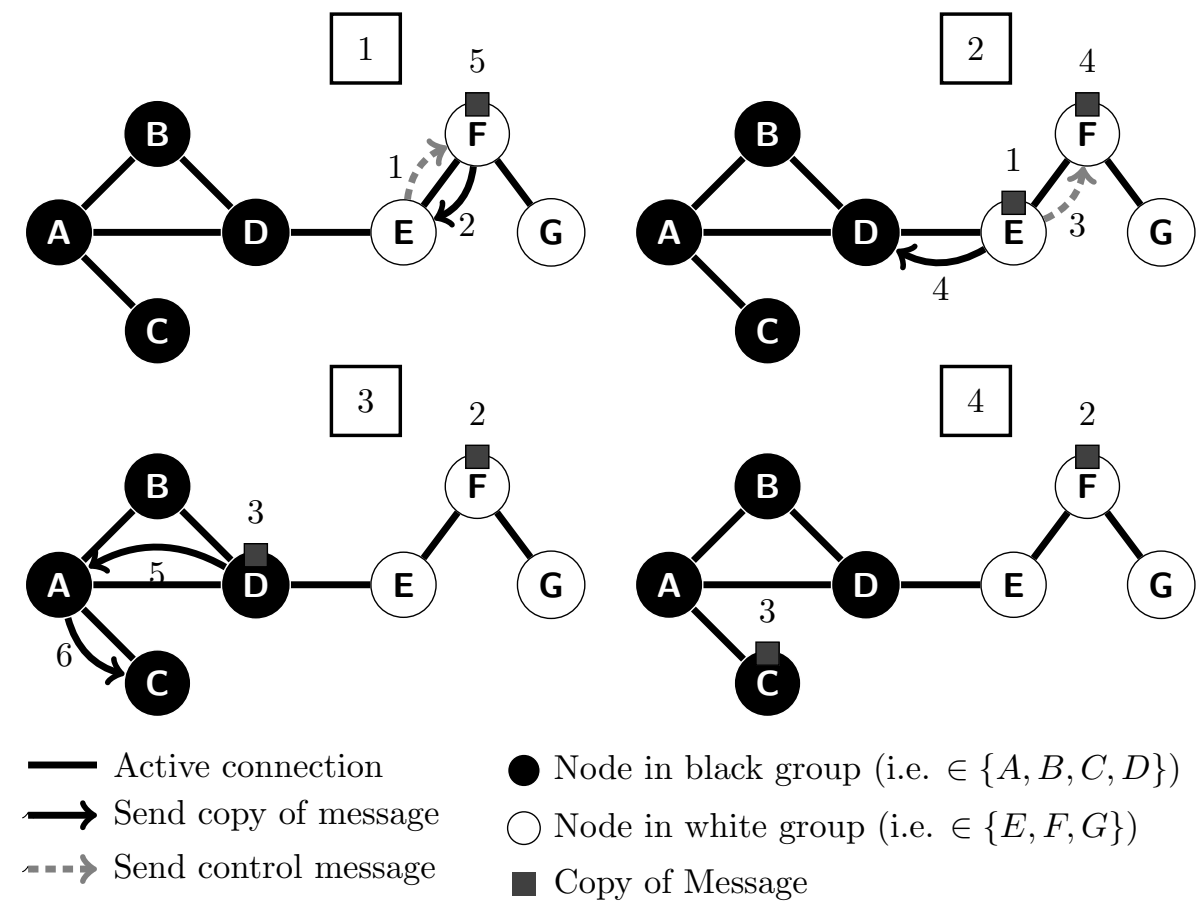

Figure 3: Example of message forwarding between two HYMAD Groups. (Full explanation in section 3.4) 
its list of held messages. Upon receiving F's broadcasted list, node E becomes aware of the new message. Being a border node, E periodically receives node D's distance vector and therefore knows that the message's destination is a member of the black group. E therefore requests (1) a copy from $\mathrm{F}$, who forwards it on (2). Before transferring the message on to D, F believes (correctly) that it is white group's only border node and thus determines that it must send 3 copies to the black group. $\mathrm{E}$ then asks $\mathrm{F}$ to reduce its number of copies by 2, to which $\mathrm{F}$ answers positively (3). Now that $\mathrm{E}$ has its 3 copies, it forwards them on to D (4). D receives these copies and realizes that they are for C. Through its intra-group routing protocol, D knows that the shortest path to $\mathrm{C}$ runs through A. It therefore transmits the message to A (5), which then forwards it to C (6) for a successful delivery.

Had C not been the final destination for the message, steps 1-4 would still have occurred, because $\mathrm{E}$ would have compared the message lists from both the black and white groups and determined that the black group did not have a copy of the message. Upon receiving the message, node D would have chosen a random custodian for the message within its own group and then forwarded the 3 copies to it.

\subsection{Discussion}

\subsubsection{Reacting to topology changes}

In the previous example, the connectivity conditions were idyllic. But what would have happened if the link between $\mathrm{D}$ and $\mathrm{E}$ had come down during the message transfer? The white group would then have had two custodians of the message, with different numbers of copies. What if the link between A and D had broken, thus extending black group's diameter to 3? Either D or C would have been excluded from it.

There are a number of situations, such as when two groups merge, where copies of a given message can be distributed among several group members. This is not a problem because everyone in the group will be aware of this situation through the Messages-in-group list broadcasts. When a border node wishes to send some copies to a neighboring group, it will consider the total number of copies in the group, request a copy from one custodian, determine how many copies to send, and then iteratively ask each custodian to provide enough copies through Reduce Number of Copies messages. Over time, the group will return to the stable situation of a unique custodian with the last remaining copy of the message for the group.

An internal link failure may increase a group's diameter, thus causing it to split into several separate sub-groups. In such a situation, each sub-group only has a fraction of the messages of the original group. Fortunately this is not really a problem. Firstly, the intra-group protocol detailed in section 3.2 ensures that nodes will update their message lists accordingly when removing nodes from their group member lists, thereby preventing a sub-group from advertising messages it does not have or any other such incoherences. Secondly, certain subgroups may still be connected to each other. If either sub-group has more 
than one copy of some messages, these will be copied over the other sub-group. In any case, HYMAD recovers gracefully from group splits.

\subsubsection{Managing the number of copies in a group}

Because a message can have several custodians within a group, the Reduce Number of Copies signals are necessary to avoid unnecessary retransmissions of the message. Indeed, it would be wasteful for a border node to request copies from each custodian. It only needs to get one complete copy, but has to make sure that the extra custodians reduce their number of copies accordingly so as not to create extra copies in the network. A subtle situation appears when several border nodes concurrently demand copies of a same message. How many should each get? We solve this issue by determining the number of copies to send at the last possible moment, right before forwarding the message to the next group. There are therefore three steps: (i) first send a Copy Request message in order to receive a complete copy, (ii) then estimate the number of copies held by the group and the number of border nodes to calculate the number $n$ of copies to forward on to the neighboring group, (iii) and finally use of the Reduce Number of Copies messages to ask the message's custodians to collectively reduce their number of copies by $n$. However, the custodians may only be able to reduce their number of copies by $m<n$ if another border node has requested copies in the meantime. In that case, $m+1$ copies are forwarded on to the neighboring group. This mechanism ensures that the number of copies of a message in the network remains constant at all times.

\subsubsection{The cost of clustering}

Group partitioning, or clustering, in a mobile ad-hoc network is considered a costly and difficult problem. In many approaches, the overhead increases with the network dynamics, a single change in topology can lead to a complete re-clustering of the network, and regular routing cannot function until the the clusters have stabilized [22].

However none of these problems arise in our approach. Indeed, since the Group algorithm messages are broadcast with a predefined network-wide period, the speed of topology changes has no effect how often these messages are broadcast. On the contrary, high dynamics leads to unstable groups, which means many singleton groups, shorter Group algorithm messages, and ultimately lower overhead. For a more thorough discussion of overhead, refer to Section 4.2 Furthermore, changes in one group's composition do not propagate to the entire network. If one or more nodes leave a given group, for example when a link goes down, they can only join neighboring groups if they do not increase those groups' diameter beyond $D_{\max }$. Otherwise they are simply rejected and remain alone. All group composition changes remain local. Finally, HYMAD does not require nodes to agree on group composition. For example, if a message is being sent through the intra-group routing protocol and an intermediary node realises that the destination is not in its group, he will simply stop the routing and add the message to his own list of held messages. In any case, HYMAD recovers gracefully from any inconsistencies. 


\subsubsection{Choosing the diameter parameter}

Choosing a diameter parameter for the group self-stabilization algorithm involves a trade-off. On the one hand, increasing it will expand each node's individual "knowledge horizon" of the actual network topology. Fewer copies will cover a larger portion of the network, which will naturally lead to faster delivery. On the other hand, this comes at the cost of increasing the convergence time of the group service. It may also slightly increase the group service's overhead (Section 4.2). Ideally, the convergence speed should be considerably faster than the speed of topology changes. In a sense, extreme mobility may fundamentally limit a node's possible knowledge of the network's topology.

If one is willing to incur the extra cost, the diameter can be set to encompass the entire network. In such a situation, HYMAD resembles a resilient MANET routing protocol using store-and-forward for message transfers. Furthermore, in many mobile wireless scenarios, there are underlying social dynamics at work which can sometimes drive nodes to gather into loose communities. $D_{\max }$ should be chosen so as to allow the expected number of members per social group to neatly fit into one self-stabilizing group.

\section{Simulation Results}

\subsection{Methodology}

We implemented HYMAD in the ONE DTN simulator [2] in a completely distributed fashion. The ONE simulator allows for testing many different parameters including buffer sizes, transmission speeds and ranges, message sizes, etc. It also implements a simple interference model which forbids a node from communicating (either receiving or sending) with two neighbors at the same time. In our default scenario (Figs. 5a, 6b, 7a), we considered 100MB buffers, $10 \mathrm{kB}$ message sizes, $100 \mathrm{kB} / \mathrm{s}$ transmission speeds and infinite TTLs. The message generator randomly creates a new message every second. In such a setup, buffer size and channel congestion would not be issues. However, lower transmission speeds could cause channel congestion. This, and how it interacts with the HYMAD overhead, will be examined in the next section.

Two types of simulations were conducted: the first uses the Random Waypoint mobility model and the second replays the Rollernet connectivity trace. For the Random Waypoint simulations, we kept the default ONE movement parameters (min speed: $0.5 \mathrm{~m} / \mathrm{s}$, max speed: $1.5 \mathrm{~m} / \mathrm{s}$, wait time: $2 \mathrm{~s}$ ) and experimented with different numbers of nodes and transmission ranges to study different densities, in order to explore the two dimensional mobile wireless network space of Fig. 1. We also made the link capacity vary in order to observe the network's behavior under congestion.

The results in this section are all averages obtained from 20 runs of a given scenario. A set of 20 different seeds were used in each run to initialize the message generators and the node mobility. Each simulation ran an extra 1000 seconds after the last message was created to give all sent messages time to arrive or expire. We calculated $95 \%$ confidence intervals for each measurement. 
Table 2: Overhead of HYMAD Control Messages

\begin{tabular}{ll} 
Message & Size (bits) \\
\hline Copy Request & 128 \\
\hline Reduce Number of Copies & 128 \\
\hline Group algorithm messages & $128 \times N_{\text {group members }}$ \\
\hline Messages-in-group list & $128 \times N_{\text {messages in group }}$ \\
\hline
\end{tabular}

These tend to be quite tight (typically \pm 0.02 ) and are not reported on this section's plots for clarity's sake.

We always compare HYMAD to both Epidemic and the regular Spray-andWait it extends. In the default scenario, there is little contention and Epidemic therefore provides an upper bound on achievable performance in terms of both delay and delivery ratio. This is not always true, particularly when the links are saturated (Fig. 5c). Spray-and-Wait provides a DTN state-of-the-art comparison.

Unless otherwise specified, we used $D_{\max }=2$ for most simulations because it ensures a very fast convergence rate and seemed to accurately reflect the size of separate connected components (small groups of friends for example), particularly during the accelerating phases of Rollernet (Section 4.5.1). Because of this, greater values of $D_{\max }$ yield only a small improvement of the delivery ratio. Interestingly, greater values of $D_{\max }$ do not noticeably increase the overhead, as discussed in the next section.

\subsection{HYMAD Overhead}

HYMAD makes use of several types of control messages. Two are periodic, the Group algorithm message and Messages-in-group list (Section 3.1), while the other two, Copy Request and Reduce Number of Copies, are spontaneous (Section 3.3). In order to estimate how large these control messages are, we assume that all node ids, messages ids, and priority scores can fit on 32 bits. For example, in ONE, a Copy Request message only needs to contain the id of the requesting node and the id of the requested message, hence 64 bits, which was "rounded up" to 128 bits. On the other hand, Messages-in-group list varies with the number of messages. The values and formulas used in the rest of this section are in Table 2 In this paper, in the context of the ONE simulator, we consider that neither Epidemic nor Spray-and-Wait incur any control overhead.

Fig. 4 plots the average link overhead depending on how fast new messages are appearing in the network. The overhead attributable to the Group algorithm messages is invariant and represents a lower asymptote for the total overhead. Indeed, even in the absence of any messages, the group partitioning algorithm would still be running. In Fig. 4a, where both the Group algorithm messages and the Messages-in-group lists are being broadcast every 2 seconds, the group algorithm overhead is negligible. In Fig. 4b, the Group algorithm message's broadcast frequency was increased. Even when broadcast every $100 \mathrm{~ms}$ the group partitioning algorithm's overhead remains very reasonable. In the 


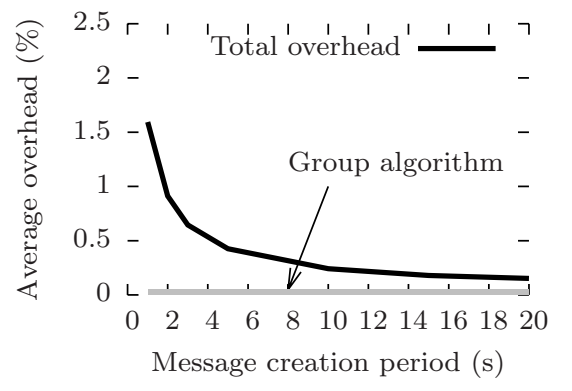

(a) $2 \mathrm{~s}$

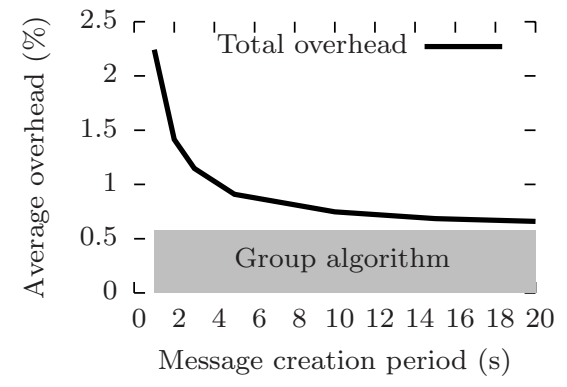

(b) $100 \mathrm{~ms}$

Figure 4: HYMAD average link overhead vs Time period of appearance of new messages in the network. The Messages-in-group list is broadcast every 2 seconds, whereas the Group algorithm messages is broadcast every 2s in Fig. 4a and every 100ms in Fig. 4b.

rest of this paper, $100 \mathrm{~ms}$ will be the default broadcasting period for the group partitioning algorithm. In both cases, the total overhead decreases non-linearly with the Messages-in-group list broadcasting period. Indeed, at greater frequencies, messages appear faster than they can be delivered. This increases the length of the Messages-in-group lists, which worsens the overhead, which in turn diminishes the link capacity available to deliver the numerous messages.

The same measurements where made by varying the HYMAD diameter parameter $\left(D_{\max }\right)$ instead of the broadcast frequency. Surprisingly, the diameter parameter has very little impact on the total overhead. Intuitively, a greater group diameter parameter should lead to larger groups, and hence larger Group algorithm messages and Messages-in-group lists, and ultimately an overhead increase. However, closer examination shows that this is not the case. Firstly, the topology limits how large groups can grow. For example, increasing your diameter to 4 if most connected components have a diameter of 2 or 3 will have little effect on overhead. Furthermore, even if the effect were noticeable (e.g. in a very dense scenario), the total overhead of the Group algorithm messages, as explained previously, would still be negligible. Secondly, lets consider a connected component that would fit either two 2-diameter groups of diameter or a single 4-diameter group. In the latter case, we expect the size of the Messages-in-group list to be proportional to the total number of messages in the 4-diameter group (i.e. the entire connected component), whereas in the former case, we would expect those control messages to be proportional to the number of messages initially held in each 2-diameter group, i.e. roughly half of those in the 4-diameter group. However the HYMAD protocol ensures that copies will be passed on between the two 2-diameter adjacent groups. Therefore, each 2-diameter group will eventually hold the same number of messages as the 4-diameter group, and their Messages-in-group lists will be exactly as long. Therefore, it follows that the diameter should have a negligible effect on total overhead.

Fig. [5 compares the performance of HYMAD, Epidemic and Spray-and-wait, 


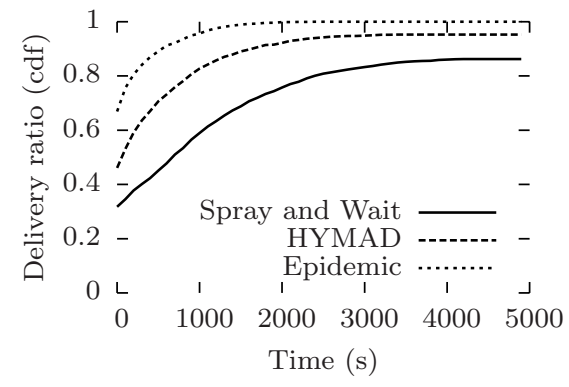

(a) $100 \mathrm{kB} / \mathrm{s}$

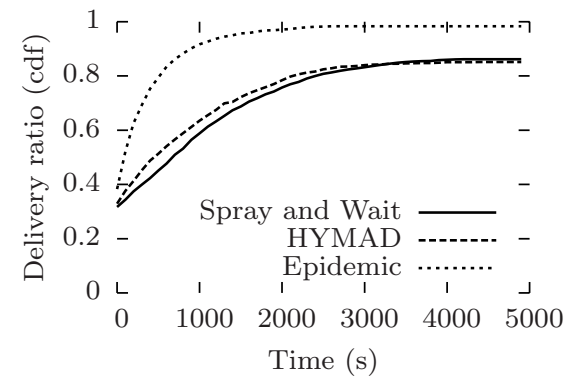

(b) $10 \mathrm{kB} / \mathrm{s}$

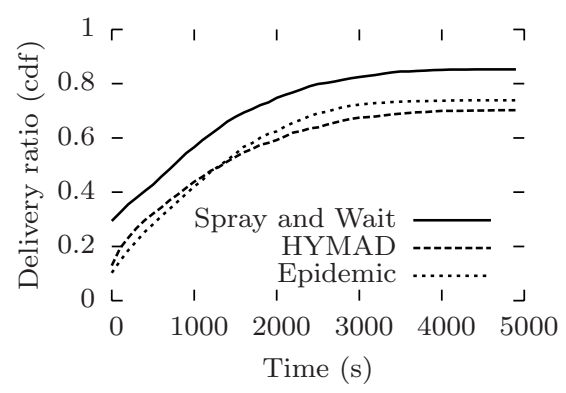

(c) $1 \mathrm{kB} / \mathrm{s}$

Figure 5: Cumulative delivery ratio using Random Waypoint with 30 nodes and varying transmission speeds.

with increasingly slow transmission speeds. Fig. $5 \mathrm{a}$ is our default scenario. When the transmission speed if divided by 10 (Fig. 5b), HYMAD's overhead and occasionally useless retransmissions (e.g. when a group splits) really start to degrade performance. When the transmission speed drops to $1 \mathrm{kB} / \mathrm{s}$, Epidemic routing's performance collapses as it saturates the links. In these very challenged conditions, Spray-and-wait actually performs the best.

All the results for HYMAD in this paper were obtained by first measuring the average per-link overhead and then subtracting that amount from the available link capacity in the ONE simulator.

\subsection{Influence of network connectivity}

Fig. 6 compares HYMAD's delivery ratio to that of both Epidemic and Spray-and-Wait for different network densities. We considered 30 nodes all moving according to the Random Wapyoint model. The only parameter that changes between these 3 scenarios is the transmission range and hence the network density.

When the network is very sparse (Fig.6a), nodes only occasionally encounter others, and rarely more than one at a time. Under such conditions, it is a good idea to distribute copies to all the nodes that one encounters, as in Spray-andWait. On the other hand, two HYMAD nodes may form a temporary two-node 


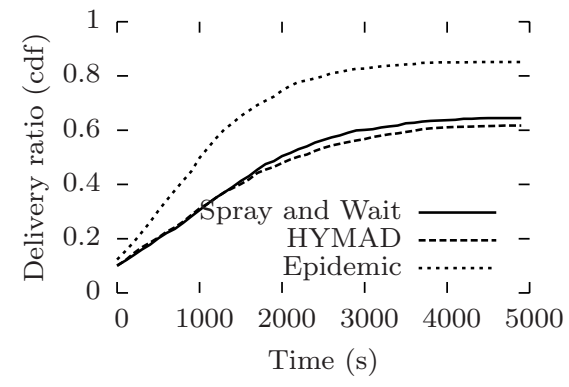

(a) Sparse $(\bar{d}=0.6 \pm 0.1, R=400 m)$

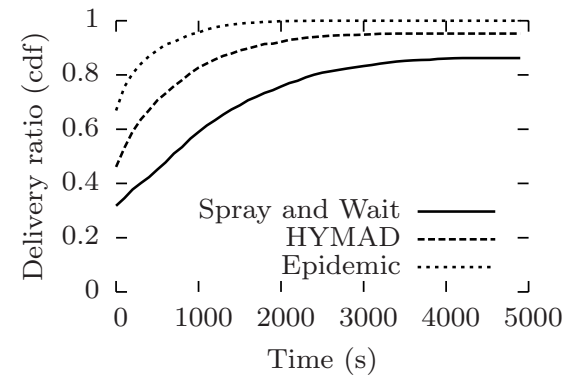

(b) Medium $(\bar{d}=1.6 \pm 0.4, R=700 m)$

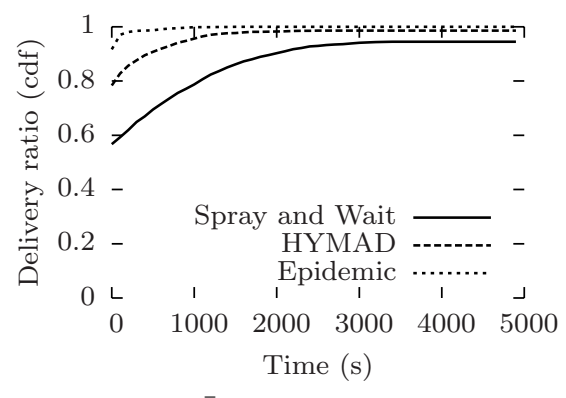

(c) Dense $(\bar{d}=6.7 \pm 0.6, R=1 \mathrm{~km})$

Figure 6: Cumulative delivery ratio using Random Waypoint with 30 nodes and varying transmission ranges (and thus varying node densities). The average node degrees $(\bar{d})$ and transmission ranges $(R)$ are indicated in parentheses

group in which neither node transmits any of its messages to the other. In the sparse case, HYMAD's group mechanisms actually degrade the delivery ratio, compared to a more straightforward protocol like Spray-and-Wait.

When the network's density increases, HYMAD's performance overtakes Spray-and-Wait's (Fig. 6b, the default scenario) to the point of nearly matching the performance of Epidemic for dense networks (Fig [6c). This is because HYMAD's knowledge of its local network topology expands with the node density. Indeed, there is a relation between the average group size, the number of copies and the number of copies necessary to cover the network quickly and efficiently. For example, in Fig. 6c, end-to-end connectivity almost always exists, and we expect $D_{\max }=2$ groups to typically contain 4 or 5 nodes out of the total 30 . Since the algorithm tends to leave a single copy per group, there is a good chance that the five copies will quickly spread to all the groups, one of which is likely to contain the destination. This allows a message to rapidly zero in on its destination and accounts for the Epidemic-like performance in the dense scenario. 


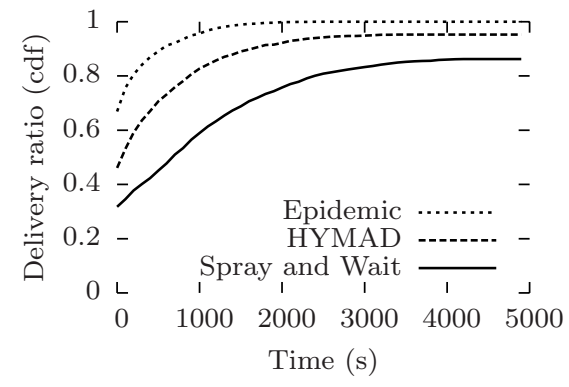

(a) 5 copies

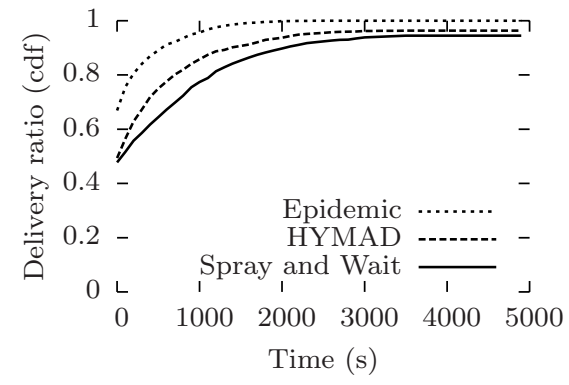

(b) 15 copies

Figure 7: Impact of the number of copies on delivery ratio

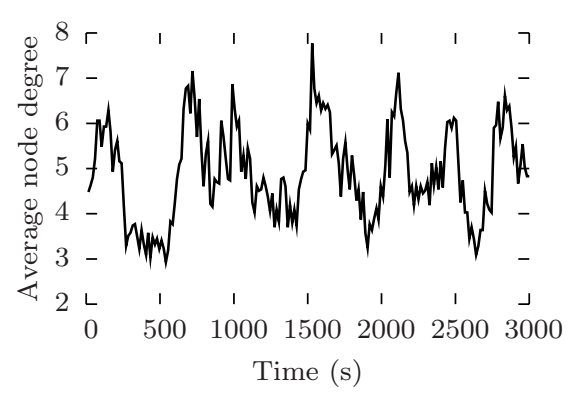

(a) Average node degree

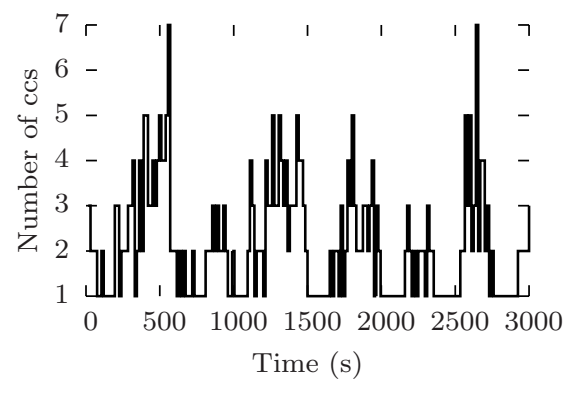

(b) Number of connected components (ccs)

Figure 8: The accordion effect in Rollernet

\subsection{Influence of the number of copies}

Fig. 7 compares the delivery ratio obtained by HYMAD and Spray-and-Wait. Fig. 7a again corresponds to our default scenario. Predictably, the performance of both Epidemic and Spray-and-Wait increases with the number of copies.

\subsection{Results on the Rollernet trace}

\subsubsection{Rollernet}

In this section, we evaluate HYMAD's performance on Rollernet [17, a highly connected and extremely mobile connectivity trace. The Rollernet experiment involved equipping 62 participants of the regular Sunday afternoon rollerblading tour through Paris with contact loggers (Intel iMotes), whose sampling period was set to 15 seconds. In order to witness different behavior profiles, the 62 bluetooth loggers were distributed among groups of friends, members of rollerblading associations, and staff operators. In particular, one member of the staff was instructed to remain behind the tour at all times while another stayed in front for the entire duration of the experiment. This allows us to get a rough sense of the relative geographic position of the participants by looking at the 
connectivity graph. A snapshot of the connectivity graph can be seen in Fig. 111 and an animation is available online [23].

The Rollernet trace is ideal for evaluating HYMAD. Indeed it exhibits the following characteristics:

- High density: Contrary to many DTN traces, Rollernet is not sparse. A look at Fig. 8a, shows that the average node degree of the connectivity graph oscillates between 2.9 and 7.8. The average for the whole tour is 4.8 .

- High mobility: Everyone eventually meets everyone else. On average, each of the 62 nodes meets 56 others during the course of the tour. Additionally the topology evolves extremely quickly. The average lifetime of a given link is 26 seconds. The average lifetime of a shortest path between two nodes is 15.5 seconds. Considering that the sampling period is 15 seconds, it follows that links are highly unstable and valid routes transient.

- Accordion Effect: This is an interesting consequence of the rollerblading context. The tour alternates between acceleration and deceleration phases in which the network topology respectively expands, leading to several separate connected component, and contracts, leading to a single connected component. Fig. 8b shows that the number of connected components varies between 1 and 7 (17 if counting isolated nodes). In fact, Figures $8 \mathrm{a}$ and $8 \mathrm{~b}$ have roughly alternating phases.

Although the accordion effect may be fairly specific to the Rollernet context, the high density and mobility are not, and the insights obtained by replaying this contact trace have a broad application to DTNs ranging from networks of hand-held mobile devices to vehicular ad-hoc networks.

\subsubsection{Group stability}

Fig. 9 compares the rate at which links come up or down in Rollernet with the number of nodes per second that either join or leave a group for $D_{\max } \in\{1,2,4\}$.

Rate of change in group composition is very steady whereas the rate of link changes follows the Rollernet accordion effect. This supports the idea that small communities like groups of friends tend to stick together during the tour and that link failures do not necessarily mean that two nodes have clearly moved away from each other. The HYMAD group structure appears to smooth the accordion effect.

Increasing $D_{\max }$ reduces the rate at which nodes join or leave groups. Indeed, even with $D_{\max }=4$, the diameter of many groups will be less than 4 . A single link failure is much less likely to increase such groups' diameter beyond $D_{\max }$ and cause nodes to leave. In this case, increasing $D_{\max }$ makes groups more stable.

\subsubsection{Performance}

To evaluate the performance of HYMAD we replayed 3000 seconds of the trace. This averages results over both the connected and disconnected phases of 


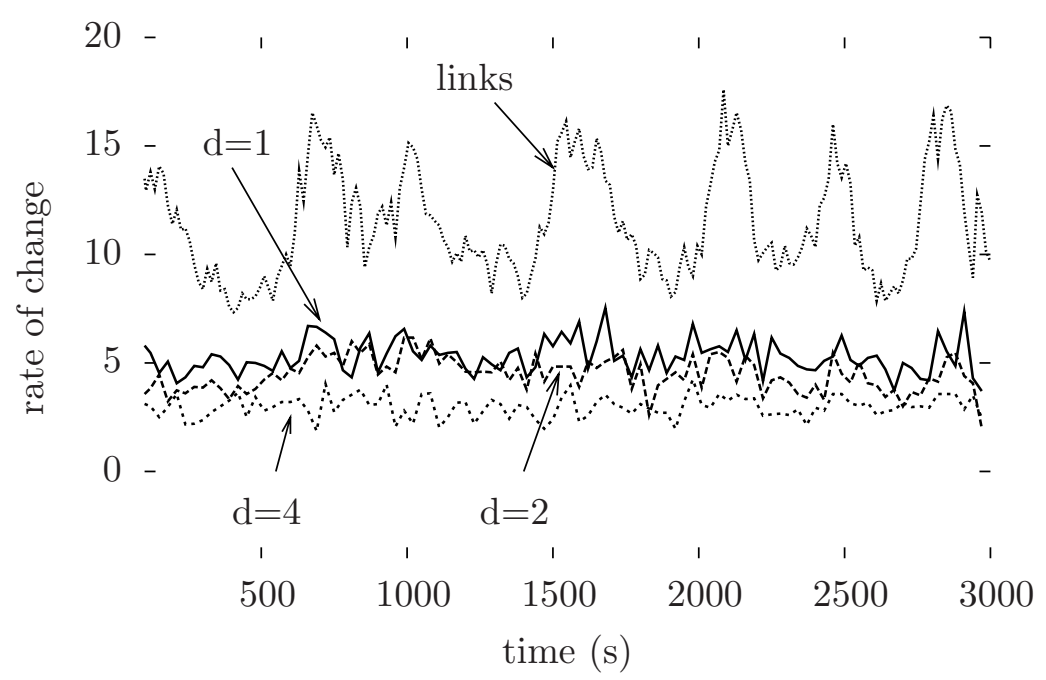

Figure 9: Link and group stability in Rollernet: number of links that come up or down per second, and number of nodes per second that either join or leave a group for varying values of $D_{\max }$.

Rollernet. Fig. 10 presents the cumulative distribution function of the delivery probability for HYMAD (with 5 copies), Spray-and-Wait (with 5 copies) and Epidemic. A few observations can be made:

- HYMAD clearly outperforms Spray-and-Wait in terms of delay and quickly achieves comparable performance with Epidemic.

- HYMAD also outperforms Spray-and-Wait in terms of delivery ratio for reasons explained hereafter.

Spray-and-Wait's simple forwarding scheme performs very well under the assumption of independent and identically distributed node mobility [16]. However this is absolutely not the case in Rollernet where groups of friends tend to stick together. It is also usually not the case in many real-world situations where underlying social dynamics are often at work.

This can have a impact on performance. For example, when using just 5 copies, Spray-and-Wait simply fails to deliver about $5 \%$ of messages even after waiting for more than 15 minutes. The average delay with Spray-and-Wait ( $210 \pm 14$ seconds) is nearly double that of HYMAD ( $120 \pm 6$ seconds). To further illustrate this point, Fig 11] compares the propagation of 10 copies after 15 seconds for HYMAD (Fig. 11a) and Spray-and-wait (Fig. 11b). The rightmost node is the head of the rollerblading tour. The bold lines represent intra-group links while the dashed gray lines represent inter-group links. The nodes holding at least one copy are represented by a diamond. In HYMAD's case, the destination is a diamond meaning that our hybrid approach has delivered its message within 15 seconds. On the other hand, the regular Spray-and-Wait protocol 


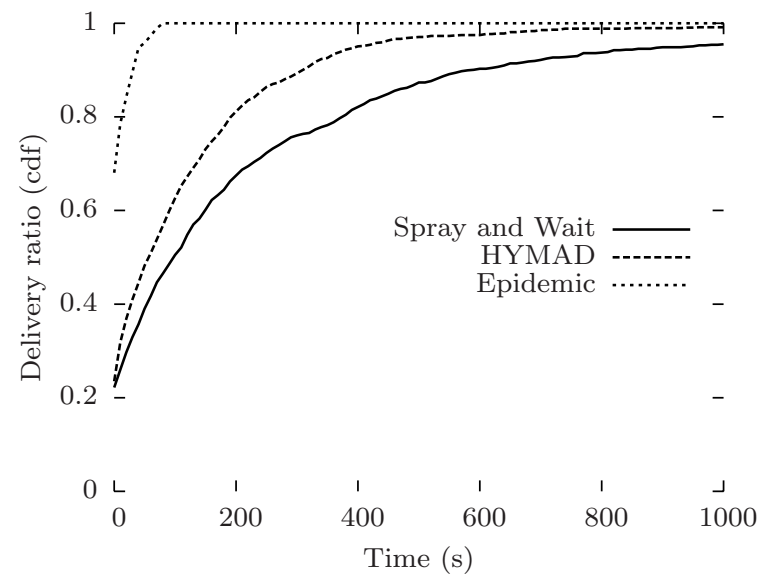

Figure 10: Rollernet: Comparison of delivery probabilities (5 copies).

distributed copies mainly within its own local group. These nodes remain close to each other thus increase the delay. In this particular case (Fig. 11b) it will take 525 seconds for a node with a copy to meet the destination

\section{Conclusion and further work}

In this paper we identified a new class of dense and highly mobile networks not well addressed by conventional DTN or MANET approaches. We proposed a new generic hybrid approach, HYMAD, that uses nodes' knowledge of their local group topology to improve the performance of a simple DTN protocol. In our case we used diameter-constrained groups along with distance vector for intra-group routing and Spray-and-Wait for inter-group routing. Simulations of our implementation in a dense and highly mobile network show significant performance improvements over regular Spray-and-Wait.

HYMAD is an example of a larger class of hybrid DTN-MANET routing protocols which can handle a very wide spectrum of networks that overlaps with those usually handled by either DTN or MANET. We believe that the first results that we obtained are encouraging for further research in this direction. In particular, does $D_{\max }$ need to be a network-wide parameter? Could it be dynamic and would nodes have to agree on it? More generally, different group partitioning algorithms would be worth exploring, while other more elaborate DTN/MANET protocol pairs could conceivably be used for intra and intergroup routing.

\section{Acknowledgments}

This work has been partially supported by the ANR project Crowd under contract ANR-08-VERS-006. 
$\diamond$ Node without any copies $\longrightarrow$ Intra-group link
$\diamond$ Node with a least one copy =--= Inter-group link

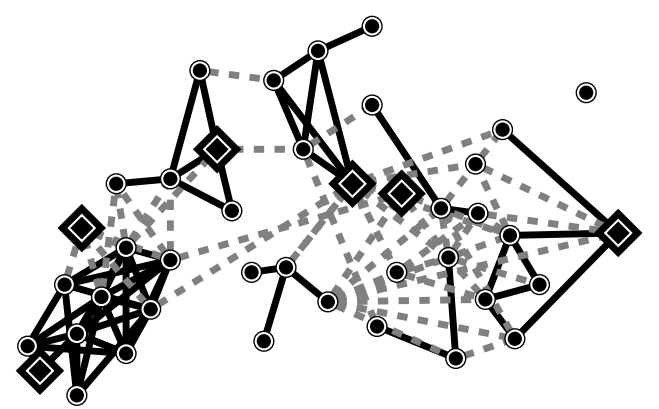

(a) HYMAD: success within 15 seconds.

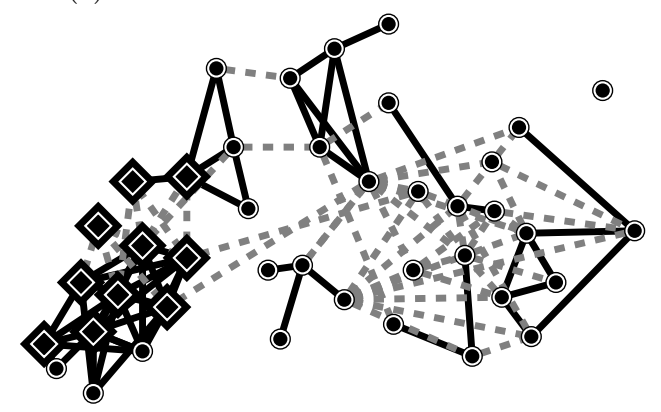

(b) Spray-and-Wait: the copies stagnate around the source. It will take a total of 525 seconds to hit the destination.

Figure 11: Regular vs Hybrid Spray and Wait routing in Rollernet. (Partial view of the topology at $\mathrm{t}=15 \mathrm{~s}$ )

\section{References}

[1] J. Whitbeck, V. Conan, HYMAD: Hybrid DTN-MANET Routing for Dense and Highly Dynamic Wireless Networks, in: AOC'09: Proceedings of the Third IEEE WoWMoM Workshop on Autonomic and Opportunistic Communications, 2009.

[2] A. Keränen, J. Ott, T. Kärkkäinen, The ONE Simulator for DTN Protocol Evaluation, in: SIMUTools '09: Proceedings of the 2nd International Conference on Simulation Tools and Techniques, 2009.

[3] L. McNamara, C. Mascolo, L. Capra, Media sharing based on colocation prediction in urban transport, in: MobiCom '08: Proceedings of the 14th ACM international conference on Mobile computing and networking, 2008.

[4] T. Claveirole, M. Boc, M. D. de Amorim, An empirical analysis of wi-fi activity in three urban scenarios, in: PERCOM '09: Proceedings of the 2009 
IEEE International Conference on Pervasive Computing and Communications, 2009.

[5] J. Harri, F. Filali, C. Bonnet, Mobility models for vehicular ad hoc networks: a survey and taxonomy, IEEE Communications Surveys \& Tutorials 11 (4) (2009) 19-41.

[6] J. Burgess, B. Gallagher, D. Jensen, B. N. Levine, MaxProp: Routing for Vehicle-Based Disruption-Tolerant Networks, in: Proc. IEEE Infocom, 2006.

[7] CRAWDAD: A community resource for archiving wirelessdata at dartmouth, http://crawdad.cs.dartmouth.edu.

[8] K. Fall, A delay-tolerant network architecture for challenged internets, in: Proc. ACM SIGCOMM, 2003.

[9] M. Grossglauser, D. N. C. Tse, Mobility increases the capacity of ad hoc wireless networks, IEEE/ACM Trans. Netw. 10 (4) (2002) 477-486.

[10] A. Lindgren, A. Doria, O. Schelen, Probabilistic routing in intermittently connected networks, in: Proc. SAPIR, 2004.

[11] E. Daly, M. Haahr, Social network analysis for routing in disconnected delay-tolerant MANETs, in: Proc. ACM MobiHoc, 2007.

[12] M. Grossglauser, M. Vetterli, Locating nodes with ease: Last encounter routing in ad hoc networks through mobility diffusion, in: Proc. IEEE Infocom, 2003.

[13] J. Ott, D. Kutscher, C. Dwertmann, Integrating dtn and manet routing, in: CHANTS '06: Proceedings of the 2006 SIGCOMM workshop on Challenged networks, 2006.

[14] S. Delaët, B. Ducourthial, S. Tixeuil, Self-Stabilizing Systems, Springer, 2005, Ch. Self-stabilization with r-Operators Revisited, pp. 68-80.

[15] B. Ducourthial, S. Khalfallah, F. Petit, Best effort group service in dynamic networks, Tech. rep. (2008).

[16] T. Spyropoulos, K. Psounis, C. Raghavendra, Spray and wait: an efficient routing scheme for intermittently connected mobile networks, in: WDTN '05: Proceedings of the 2005 ACM SIGCOMM workshop on Delay-tolerant networking, 2005.

[17] P.-U. Tournoux, J. Leguay, F. Benbadis, V. Conan, M. D. de Amorim, J. Whitbeck, The accordion phenomenon: Analysis, characterization, and impact on dtn routing, in: Proc. IEEE Infocom, 2009. 
[18] D. Antonellis, A. Mansy, K. Psounis, M. H. Ammar, Towards distributed network classification for mobile ad hoc networks, in: WICON '08: Proceedings of the 4th Annual International Conference on Wireless Internet, 2008 .

[19] V. Borrel, M. H. Ammar, E. W. Zegura., Understanding the wireless and mobile network space: a routing-centered classification, in: CHANTS '07: Proceedings of the second ACM workshop on Challenged networks, 2007.

[20] W. Zhao, M. Ammar, E. Zegura, A message ferrying approach for data delivery in sparse mobile ad hoc networks, in: MobiHoc '04: Proceedings of the 5th ACM international symposium on Mobile ad hoc networking and computing, 2004.

[21] R. C. Shah, S. Roy, S. Jain, W. Brunette, Data mules: Modeling a threetier architecture for sparse sensor networks, in: IPSN 2008: Proceedings of the First IEEE Workshop on Sensor Network Protocols and Applications, 2003.

[22] J. Yu, P. Chong, A survey of clustering schemes for mobile ad hoc networks, Communications Surveys \& Tutorials, IEEE 7 (1) (2005) 32-48.

[23] J. Whitbeck, Animation of rollernet connectivity graph, http://www youtube $\cdot$ com/watch?v=kdkCx1xlMkI 